\title{
Cyclopamine is a novel Hedgehog signaling inhibitor with significant anti-proliferative, anti-invasive and anti-estrogenic potency in human breast cancer cells
}

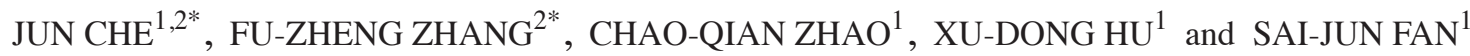 \\ ${ }^{1}$ Key Laboratory of Radiation Biology, School of Radiation Medicine and Protection, \\ Medical College of Soochow University, Suzhou, Jiangsu 215123; ${ }^{2}$ Department of Radiation Oncology, \\ The Fourth Hospital Affiliated to Soochow University, Wuxi, Jiangsu 214062, P.R. China
}

Received November 26, 2012; Accepted February 4, 2013

DOI: 10.3892/ol.2013.1195

\begin{abstract}
Stimulation of Hedgehog (Hh) signaling induces carcinogenesis or promotes cell survival in cancers of multiple organs. In epithelial cancer with aberrant Hedgehog activation, abrogation of Hedgehog signaling by cyclopamine, a naturally occurring Hedgehog-specific small-molecule inhibitor, causes profound inhibition of tumor growth. In the present study, cyclopamine displayed a significant potency in suppressing the proliferation of both estrogen-responsive (MCF-7) and estrogen-independent (MDA-MB-231) human breast cancer cells. Cyclopamine induced a robust $\mathrm{G} 1$ cell cycle arrest and elicited notable effects on the expression of cyclin D1 through modulation of the MAPK/ERK signaling pathway. Cyclopamine also inhibited the invasive ability of both breast cancer cell lines by suppressing the expression

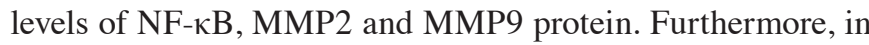
estrogen-responsive MCF-7 cells, cyclopamine significantly downregulated the production of estrogen receptor- $\alpha$ protein. Our results implicate cyclopamine as a novel, potent inhibitor of human breast cancer proliferation and estrogen responsiveness that could potentially be developed into a promising therapeutic agent for the treatment of breast cancer.
\end{abstract}

\section{Introduction}

It was anticipated that by 2010 , breast cancer would be newly diagnosed in $>1.5$ million females each year, and that 500,000 females worldwide would succumb to this disease (1).

Correspondence to: Professor Sai-Jun Fan, Key Laboratory of Radiation Biology, School of Radiation Medicine and Protection, Medical College of Soochow University, 199 Renai Road, Suzhou, Jiangsu 215123, P.R. China

E-mail: sjfan@suda.edu.cn

*Contributed equally

Key words: cyclopamine, breast cancer, MAPK/ERK, estrogen receptor- $\alpha$, proliferation, invasion
Therapies that target the drivers of individual types of breast cancer have substantially improved the outcome of females with breast cancer $(2,3)$.

One such pathway is the hedgehog (Hh) signaling pathway, which specifies patterns of cell growth and differentiation during embryogenesis in a wide range of tissues (4). In addition to its role in developmental patterning, this pathway plays a critical role in mature tissue homeostasis and the maintenance of somatic cell numbers in various organs (5). Aberrant activation of the Hh pathway is common in breast carcinoma (6). The Hh pathway represents an attractive target for drug development and has demonstrated potential in clinical trials of cancer treatments. The specificity of cyclopamine for the $\mathrm{Hh}$ pathway is demonstrated by an absence of cytotoxicity in cells that lack Hh signaling (6).

In the present study, using estrogen-responsive (MCF-7) and estrogen-independent (MDA-MB-231) human breast cancer cell lines, the effects of cyclopamine on breast cancer proliferation and invasion are investigated in vitro. The possible pathways involving its regulation of breast cancer tumorigenesis and metastasis are explored.

\section{Materials and methods}

Cell culture and reagents. The MCF-7 and MDA-MB-231 human breast cancer cell lines were purchased from the American Type Culture Collection (Manassas, VA, USA). Cells were maintained in Dulbecco's modified Eagle's medium supplemented with $10 \%$ fetal calf serum, L-glutamine (5 mmol/l), non-essential amino acids $(5 \mathrm{mmol} / \mathrm{l})$, penicillin $(100 \mathrm{U} / \mathrm{ml})$ and streptomycin $(100 \mathrm{U} / \mathrm{ml})$ (Invitrogen Life Technologies, Carlsbad, CA, USA), at $37^{\circ} \mathrm{C}$ in a humidified atmosphere at $5 \% \mathrm{CO}_{2}$. Cyclopamine and the MEK1/2 inhibitor, U0126, were obtained from Cell Signaling Technology, Inc. (Beverly, MA, USA).

The study was approved by the Ethics Committee of Soochow University, Suzhou, Jiangsu, China.

Cell viability assay. Cell proliferation was determined using an MTT viability assay; the most commonly used assay for determining cell growth and death. The MTT survival assay 


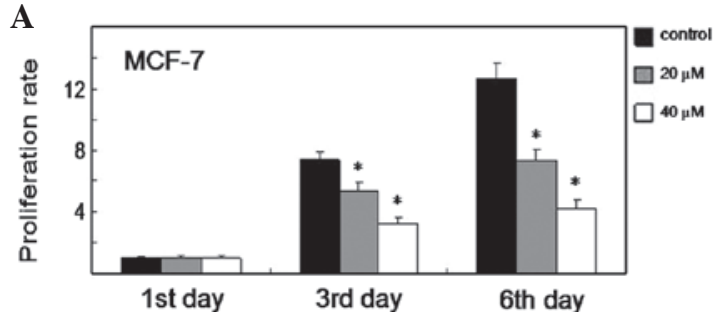

C

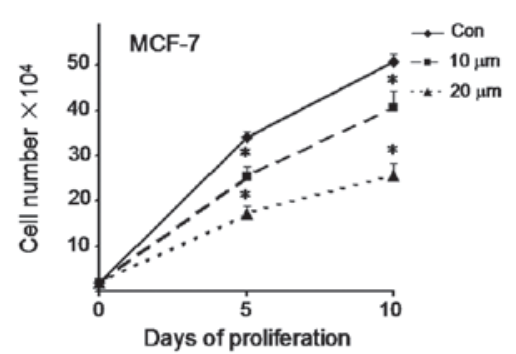

B

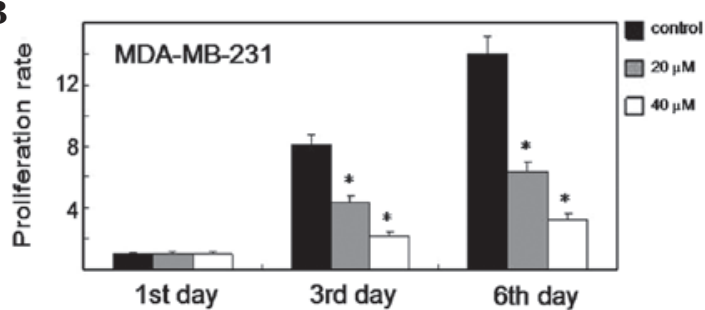

D

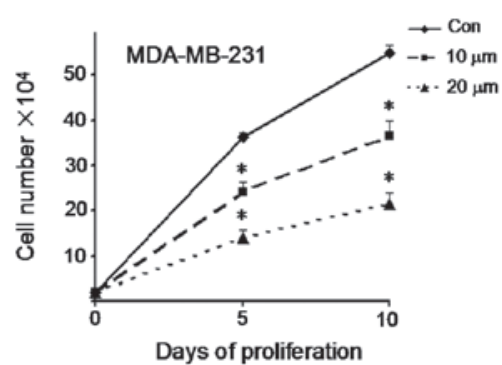

Figure 1. Cyclopamine decreases the proliferation of human breast cancer cells. (A and B) Breast cancer cells were treated with or without cyclopamine for 6 days, and then counted at the indicated time. All samples were prepared in triplicate. The proliferation rate was measured as fold changes in cell growth. (C and D) Proliferation curve of breast cancer cells. Cells were treated with cyclopamine and counted after 5 and 10 days. Results are representative of three independent experiments.

has been described in detail previously (7). Exponentially growing cells were recultured (5,000 cells/well) overnight in 96-well tissue culture plates. Up to $20 \mu 1$ MTT (Sigma-Aldrich, St Louis, MO, USA) was directly added to the media in each well, at a final concentration of $2 \mathrm{mg} / \mathrm{ml}$. Following $4 \mathrm{~h}$ of incubation, the medium containing MTT was discarded, and $120 \mu 1$ dimethyl sulfoxide was added for $10 \mathrm{~min}$. The absorbance was measured using an enzyme-linked immunosorbent assay reader at $570 \mathrm{~nm}$, with the absorbance at $630 \mathrm{~nm}$ as the background correction. The cell viability was expressed as the percentage of untreated controls. All experiments were performed at least three times.

Proliferation assay. Cells were counted and plated at the same initial density on 6-well plates. They were then treated with 10 or $20 \mu \mathrm{mol} / 1$ cyclopamine or the vehicle only, and incubated for time periods ranging from 0-10 days. At each time point, cells were trypsinized and counted using a Neubauer hemocytometer under trypan blue exclusion.

Cell cycle assays. The cells were removed with trypsin and collected in centrifuge tubes together with the culture medium. The contents were centrifuged for $5 \mathrm{~min}$ at $1,800 \mathrm{x} \mathrm{g}$. The supernatant was poured out, washed once with $1 \mathrm{X}$ phosphate-buffered saline (PBS) and centrifuged for a further $5 \mathrm{~min}$. The cells were fixed with $5 \mathrm{ml}$ of pre-cooled $70 \%$ ethanol for $\geq 4 \mathrm{~h}$. The fixed cells were centrifuged and washed with $1 \mathrm{X}$ PBS. Following centrifugation, the cell pellets were resuspended in $500 \mu \mathrm{l}$ propidium iodide $(10 \mu \mathrm{g} / \mathrm{ml})$ containing $300 \mu \mathrm{g} / \mathrm{ml}$ RNase (Sigma-Aldrich). Subsequently, the cells were incubated on ice for $30 \mathrm{~min}$, and then filtered with a 53- $\mu \mathrm{m}$ nylon mesh. The cell cycle distribution was calculated from 10,000 cells with ModFit LT software (Becton Dickinson, San José, CA, USA) using FACSCalibur (Becton Dickinson).

Transwell invasion assay. The invasion assay was carried out using Transwell plates (Millipore, Billerica, MA, USA), as previously described (8). The filter surfaces (pore size, $8 \mu \mathrm{m}$ ) of the Transwell plates were uniformly coated with $25 \mathrm{mg}$ Matrigel (Becton Dickinson, San Jose, CA, USA) overnight at $4^{\circ} \mathrm{C}$, prior to the experiment. The lower chamber was filled with culture medium containing $10 \%$ fetal calf serum. The subconfluent proliferating cells were carefully transferred onto the coated upper surface of the chamber. Following $24 \mathrm{~h}$ of incubation, the filter was gently removed and the upper surface was wiped to remove all attached cells. The cells that had invaded through the Matrigel and attached to the lower surface of the filter were fixed with $4 \%$ paraformaldehyde and stained with Giemsa (SigmaAldrich, St Louis, MO, USA). Three replicates were conducted for each condition, and 15 random fields in each replicate were selected and counted using an Olympus CKX41 inverted microscope (Olympus, Tokyo, Japan). The results are presented as the ratio of the cells that invaded under experimental conditions relative to those that invaded under control conditions.

Western blot analysis. Cell lysates were prepared and western blot analysis was performed as previously described (9). Equal aliquots of total cell protein $(50 \mu \mathrm{g} / \mathrm{lane})$ were electrophoresed on sodium dodecyl sulfate (SDS)-polyacrylamide gels, transferred onto polyvinylidene fluoride (PVDF) membranes and then blotted using the following primary antibodies (1:1,000 dilution; Santa Cruz Biotechnology, Inc., Santa Cruz, CA, USA): $\beta$-actin (C-4), NF-кB (P65A), cyclin D1 (A-12), MMP2 (2C1), MMP9 (6-6B); along with secondary antibody horseradish peroxidase-labeled goat anti-mouse (GAM-007) and goat anti-rabbit (SC-2004) IgG. The protein bands were visualized using an enhanced chemiluminescence system (Union Bioscience Corporation, Hangzhou, China) with prestained markers as molecular size standards. The densitometry of the protein bands was quantified with Quantity One (Bio-Rad, Hercules, CA, USA), and the values were expressed relative to $\beta$-actin (the control for loading and transfer). At least three independent experiments were performed for each cell type studied. 
A

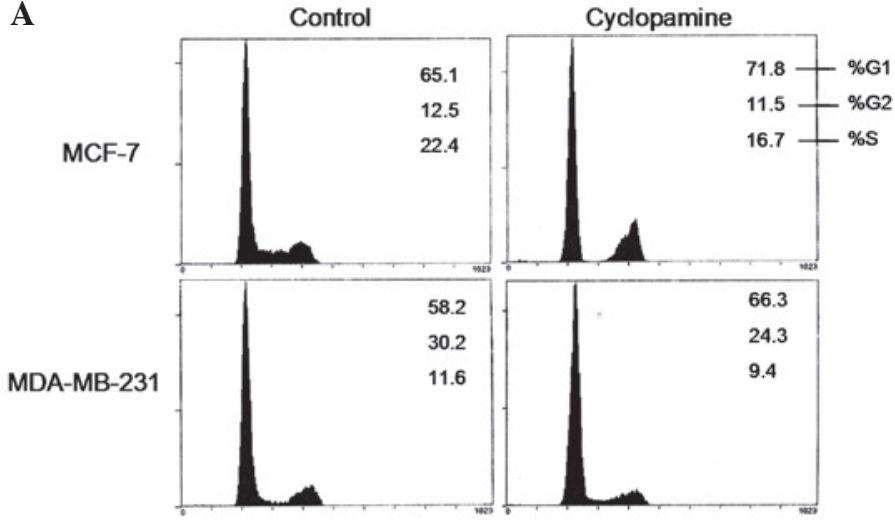

B

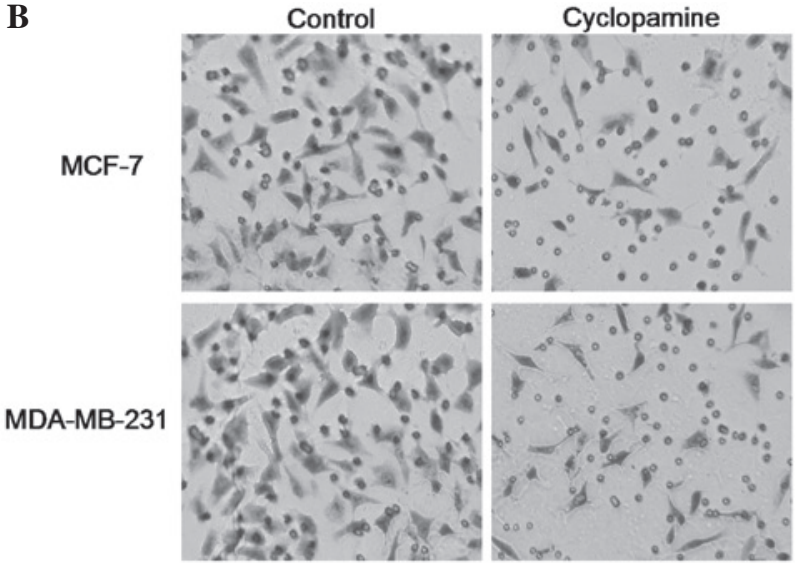

Figure 2. Cyclopamine affects breast cancer cell cycle and invasive ability. (A) Cell cycle distribution was evaluated by propidium iodide labeling and analysed by flow cytometry. (B) Cells treated with or without cyclopamine were trypsinized and transferred to the upper compartment of the modified Transwell chambers. Following $24 \mathrm{~h}$ of incubation, the invasive cells attached to the lower surface of the Matrigel-coated filter were fixed, stained and photographed under a phase contrast microscope and then counted in 15 randomly selected microscopic fields.

A

\section{MCF-7}

Dose of cyclopamine $(\mu \mathrm{M})$

\begin{tabular}{lll}
\hline Con $\quad 20 \quad 40$
\end{tabular}
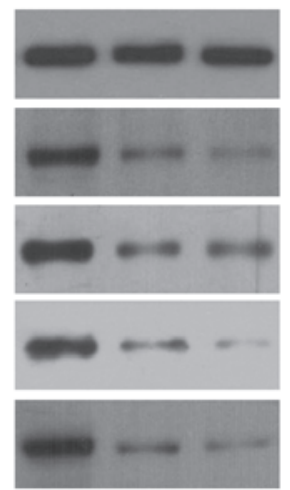

$\beta$-actin
$(42 \mathrm{kDa})$
$\mathrm{NF}-\mathrm{kB}$
$(65 \mathrm{kDa})$
Cyclin D1
(34 kDa)
MMP2
(72 kDa)
MMP9
(92 kDa)

MDA-MB-231
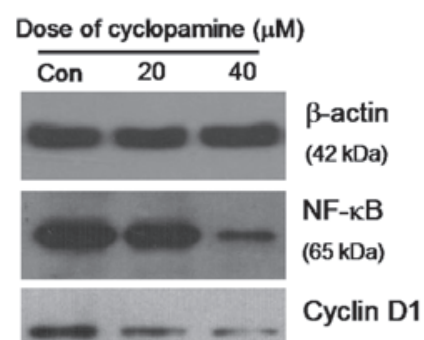

(34 kDa)

MMP2

(72 kDa)

MMP9

(92 kDa)
B

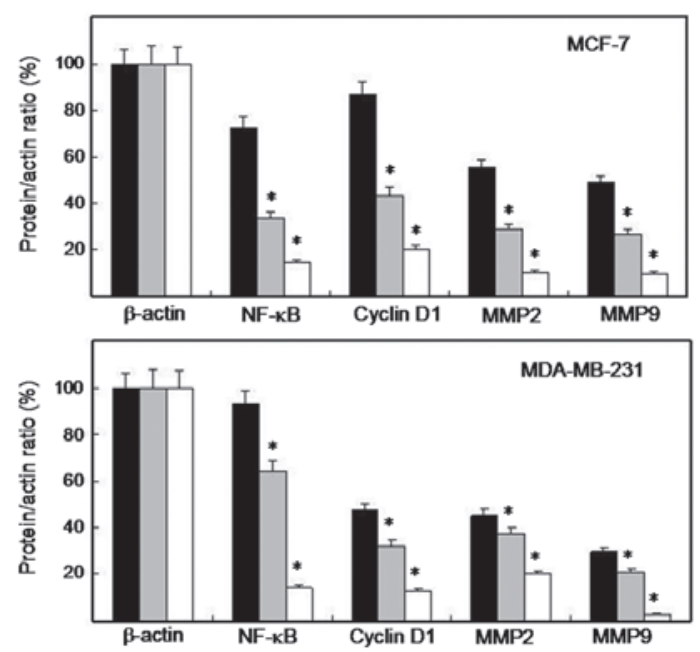

Figure 3. Cyclopamine inhibits the expression of proliferation and invasion-related proteins. (A) Whole cell lysates were prepared, and $50 \mu \mathrm{g}$ proteins were resolved using SDS-PAGE, followed by immunoblotting with the indicated specific antibodies against NF- $\mathrm{kB}$, cyclin D1, MMP2 and MMP9. (B) The expression levels are displayed as fold changes in band density. ${ }^{*} \mathrm{P}<0.01$ vs. the control group.

\section{Results}

Cyclopamine decreases breast cancer cell proliferation. MTT viability assays were conducted to elucidate the potential biological effects of cyclopamine in breast cancer cells. As shown in Fig. 1A, the MCF-7 cells treated with cyclopamine displayed a significant reduction in proliferation rate compared with the control cells on days 3 and $6(\mathrm{P}<0.01)$. Significantly, cyclopamine demonstrated the same effect in MDA-MB-231 cells (Fig. 1B) $(\mathrm{P}<0.01)$. We also tested the effect of cyclopamine on cell proliferation using an alternative method. MCF-7 cells were treated with cyclopamine (10 and $20 \mu \mathrm{M})$ or the vehicle only and incubated for time periods ranging from 0-10 days (Fig. 1C). At each time point, the cells were trypsinized and counted using a Neubauer hemocytometer under trypan blue exclusion. Cyclopamine was observed to induce a significant decrease in cell proliferation 5 and 10 days later $(\mathrm{P}<0.01)$. Notably, cyclopamine demonstrated the same effect in MDA-MB-231 cells (Fig. 1D) $(\mathrm{P}<0.01)$. The results imply that cyclopamine plays a key role in the growth control of breast cancer cells.

Cyclopamine induces G1 cell cycle arrestand inhibits theinvasive ability of both estrogen-responsive and non-responsive human breast cancer cells. Cell cycle analysis was then performed on the regulation of cyclopamine in breast cancer cells. The results in Fig. 2A suggest that cyclopamine significantly induced cell accumulation in the $\mathrm{G} 1$ phase $(\mathrm{P}<0.01)$ and a modest decrease in the $\mathrm{S}$ population percentage, from 22 to $16 \%(\mathrm{P}<0.01)$ in the MCF-7 cells. Moreover, cyclopamine also caused a significant increase in $\mathrm{G} 1$ cells in the MDA-MB-231 cells. 
A

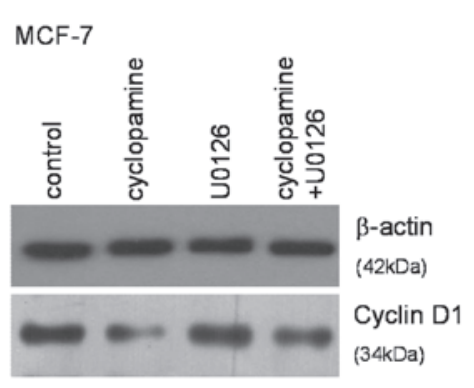

C

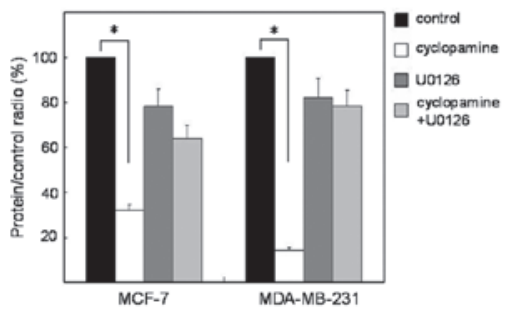

B

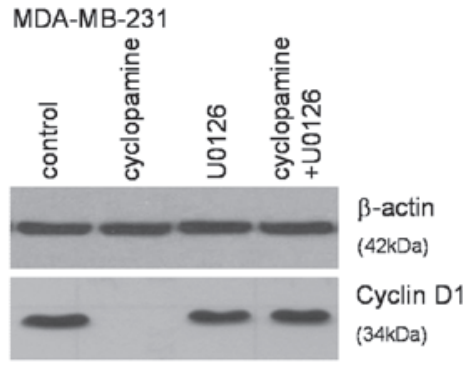

D

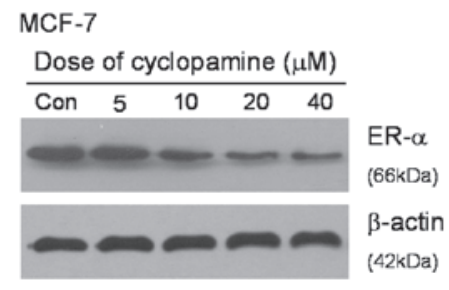

Figure 4. U0126 partly rescues cyclin D1 inhibition to cyclopamine treatment. (A and B) Comparison of cyclin D1 expression between cells treated with or without cyclopamine. (C) Statistical analysis was conducted using a Student's t-test. The expression levels are displayed as fold changes in band density. ${ }^{*} \mathrm{P}<0.01$ vs. the control group. (D) Dose-dependent effects of cyclopamine on expression of estrogen receptor- $\alpha$ (ER- $\alpha$ ) protein. MCF-7 cells were treated with indicated concentrations of cyclopamine for $24 \mathrm{~h}$, and the level of ER- $\alpha$ was determined by western blot analysis.

A critical event in tumor metastasis and progression is the ability of tumor cells to invade the extracellular matrix, allowing the tumor cells to move beyond the restrictions of the primary tumor environment. To examine the competency of cells to invade through biological matrices in vitro, a Transwell assay was conducted as described previously. The results demonstrated that, compared with the control cells, cyclopamine vigorously inhibited the ability of the MCF-7 and MDA-MB-231 cells to invade through the filter coated with Matrigel. As shown in Fig. 2B, the invasion rate of cyclopamine-treated cells significantly decreased compared with the control cells.

Cyclopamine affected the expression level of cell cycle- and invasion-related proteins. The alterations in the cell cycleand invasion-related proteins in cyclopamine-treated cells were evaluated and compared with the control cells using western blot analysis. Fig. 3A shows that the expression level of cyclin D1 decreased in cyclopamine-treated cells. The expression levels of NF- $\kappa \mathrm{B}, \mathrm{MMP} 2$ and MMP9 were also suppressed. The densitometry of the protein bands was quantified with Quantity One, and the values were expressed relative to $\beta$-actin. As shown in Fig. 3B, the expression level of the four proteins significantly decreased $(\mathrm{P}<0.01)$.

Cyclopamine modulates the expression of cyclin D1, via the MAPK/ERK signaling pathway, and downregulates the expression of $E R$ - $\alpha$. Western blot analysis was utilized to identify the targets of the Hedgehog signal pathway that may be involved in suppressing proliferation. The expression of cyclin D1 is a key initial response to cell cycle distribution and proliferation. The specific signaling cascade involved in this response was explored using a MEK1/2 inhibitor (U0126) specific to the MAPK/ERK pathway. The results showed that cyclopamine significantly inhibited the expression of cyclin D1 in both MCF-7 and MDA-MB-231 cells. To certify the potential effect of MAPK/ERK in response to cyclopamine treatment, the two cell lines were treated with U0126 prior to cyclopamine treatment. The results demonstrated that U0126 could partially prevent cells from cyclopamine-induced cyclin D1 inhibition (Fig.4A and B). The reduced cyclin D1 expression in cyclopamine-treated cells was inhibited by the MEK1/2 inhibitor (Fig. 4C), which suggested that cyclopamine mediated the expression of cyclin D1 through modulating the MAPK/ERK pathway.

The underlying mechanism for the ability of the majority of anticancer drugs to cause a growth arrest of breast cancer, is that the drugs downregulate the expression of ER- $\alpha$. To determine whether cyclopamine has a similar effect on ER- $\alpha$ production, MCF-7 human breast cancer cells were treated with a range of concentrations of cyclopamine and the level of total ER- $\alpha$ protein was monitored by western blot analysis of total cell extracts. As shown in Fig. 4D, cyclopamine strongly downregulated ER- $\alpha$ levels, and $\beta$-actin was used as a constitutive gel loading control.

\section{Disscusion}

Breast cancer is the second leading cause of cancer-related mortality in females worldwide. Alternative strategies are required to combat the deaths caused by this disease. Chemotherapy, radiation, surgery and immunotherapy are among the current treatment options for breast cancer. Chemotherapy using synthetic compounds, although demonstrated to be effective in cancer treatment, also induces severe side effects due to their toxicity in non-cancerous tissues (10). In recent years, therapeutic strategies that specifically target aberrant signaling pathways in metastatic breast cancer greatly enhance survival, while at the same time reducing bystander toxicity in non-tumor tissues (11). 
The MAPK pathway plays an important role in regulating a number of downstream molecules including kinases and scaffold proteins (12). The balance between these molecules exerts cellular responses, including cell proliferation, cell cycle arrest, migration and differentiation. Extracellular signal-regulated kinases are members of the MAPK family that transduce signals from various environmental stresses, growth factors and steroid hormones (13).

The present study has identified that cyclopamine has potent antiproliferative properties as a potential therapeutic agent for the treatment of human breast cancers by suppressing MAPK/ERK-mediated signaling. Our results show that cyclopamine significantly increased the potency of the cell cycle arrest in both human estrogen-responsive and estrogen-independent human breast cancer cells, which suggests that it may potentially be used for treating a wide range of breast cancer types, which has been a fundamental problem with the available therapeutic compounds.

Furthermore, cyclopamine inhibits the expression of ER- $\alpha$, which mediates the mitogenic properties of estrogens, and acts with tamoxifen to inhibit the proliferation of steroid-responsive MCF-7 human breast cancer cells to a greater extent than either compound alone. This result implicates the use of cyclopamine in combination with anti-estrogen therapies that would allow lower doses and thereby reduced side effects or decreased resistance to the anti-estrogens.

\section{Acknowledgements}

This study was supported by grants from the Program for Changjiang Scholars and Innovative Research Team in University (IRT0849) and the Priority Academic Program Development of Jiangsu Higher Education Institutions (PAPD).

\section{References}

1. Ginsburg OM and Love RR: Breast cancer: a neglected disease for the majority of affected women worldwide. Breast J 3: 289-295, 2011.

2. McDermott SP and Wicha MS: Targeting breast cancer stem cells. Mol Oncol 5: 404-419, 2010.

3. Laxmi Y, Elegbedea JA and Carpera SW: Methyl jasmonate decreases membrane fluidity and induces apoptosis via tumor necrosis factor receptor 1 in breast cancer cells. Anticancer Drugs 8: 766-776, 2008

4. Shibani M, Natalya F, Andrea S, et al: Hedgehog signaling and response to cyclopamine differ in epithelial and stromal cells in benign breast and breast cancer. Cancer Biol Ther 6: 674-683, 2006.

5. Kumara S, Indrajit R, Anchooria RK, et al: Targeted inhibition of hedgehog signaling by cyclopamine prodrugs for advanced prostate cancer. Bioorg Med Chem 6: 2764-2768, 2008.

6. Bara EE, Chaudhrya A, Lina A, et al: Cyclopamine-mediated hedgehog pathway inhibition depletes stem-like cancer cells in glioblastoma. Stem Cells 10: 2524-2533, 2007.

7. Li XL, Meng QH, Fan SJ, et al: Adenovirus-mediated expression of UHRF1 reduces the radiosensitivity of cervical cancer HeLa cells to gamma-irradiation. Acta Pharmacol Sin 4: 458-466, 2009.

8. Jiao Y, Ge CM, Meng QH, et al: Adenovirus-mediated expression of Tob1 sensitizes breast cancer cells to ionizing radiation. Acta Pharmacol Sin 10: 1628-1636, 2007.

9. Jiao Y, Ge CM, Meng QH, et al: Dihydroartemisinin is an inhibitor of ovarian cancer cell growth. Acta Pharmacol Sin 7: 1045-1056, 2007.

10. Somesh B and Alahari SK: miRNA control of tumor cell invasion and metastasis. Int J Cancer 6: 1283-1290, 2010.

11. Nguyena $\mathrm{HH}$, Lavrenove $\mathrm{SN}$, Sundara $\mathrm{SN}$, et al: 1-Benzyl-indole-3-carbinol is a novel indole-3-carbinol derivative with significantly enhanced potency of anti-proliferative and anti-estrogenic properties in human breast cancer cells. Chem Biol Interact 3: 255-266, 2010.

12. Chen L, Mayer JA, Krisko TI, et al: Inhibition of the p38 kinase suppresses the proliferation of human ER-negative breast cancer cells. Cancer Res 23: 8853-8861, 2009.

13. Li MW, Mruk DD, Cheng CY, et al: Mitogen-activated protein kinases in male reproductive function. Trends Mol Med 15: 159-168, 2009. 